Toro-Zapata, H.D., \& Olivar-Tost, G. (2018). Mathematical model for the evolutionary dynamics of innovation in city public transport systems. Copernican Journal of Finance \& Accounting, 7(2), 77-98. http://dx.doi.org/10.12775/CJFA.2018.010

\author{
Hernán Darío Toro-Zapata* \\ Universidad del Quindío \\ Universidad Nacional de Colombia - Manizales \\ Gerard Olivar-Tost** \\ Universidad Nacional de Colombia - Manizales
}

\title{
MATHEMATICAL MODEL FOR THE EVOLUTIONARY DYNAMICS OF INNOVATION IN CITY PUBLIC TRANSPORT SYSTEMS
}

Keywords: technological change, public transportation investment, public transportation, simulation modeling.

J E L Classification: 033, C630, R480, R420.

Abstract: In this study, a mathematical model is formulated and studied from the per-
spective of adaptive dynamics (evolutionary processes), in order to describe the inte-
raction dynamics between two city public transport systems: one of which is establi-
shed and one of which is innovative. Each system is to be influenced by a characteristic
attribute; in this case, the number of assumed passengers per unit it that can transport.
The model considers the proportion of users in each transport system, as well as the
proportion of the budget destined for their expansion among new users, to be state va-
riables. Model analysis allows for the determination of the conditions under which an

Date of submission: May 25, 2018; date of acceptance: August 2, 2018.

* Contact information: hdtoro@uniquindio.edu.co, Universidad del Quindío, Facultad de Educación, Licenciatura en Matemáticas, bloque F1 Tercer Piso, Armenia, Quindío, Colombia, phone: +57 (6) 7359382; ORCID ID: https://orcid.org/0000-00015519-9182.

** Contact information: golivart@unal.edu.co, Universidad Nacional de Colombia, Departamento de Matemáticas y Estadística, bloque y Piso 2. Manizales, Caldas, Colombia, phone: +57 (6) 8879400 ext. 55734; ORCID ID: https://orcid.org/0000-0003-1862-4842. 
innovative transportation system can expand and establish itself in a market which is initially dominated by an established transport system. Through use of the adaptive dynamics framework, the expected long-term behavior of the characteristic attribute which defines transport systems is examined. This long-term study allows for the establishment of the conditions under which certain values of the characteristic attribute configure coexistence, divergence, or both kinds of scenarios. The latter case is reported as the occurrence of evolutionary ramifications, conditions that guarantee the viability of an innovative transport system. Consequently, this phenomenon is referred to as the origin of diversity.

\section{IIIINTRODUCTION}

The city of Bogotá, Colombia is on the cusp of becoming one of the new world megacities. While in 1960, only seven megacities existed, by 2010, this number had increased to 27 , and by 2020 , it is projected that this number will grow to 37. In this growth process, cities cannot ignore fundamental aspects of their own economic and demographic development, or the complex network of interactions generated thereby (Kennedy, Stewart, Ibrahim, Facchini \& Mele, 2014, p. 7; Baccini \& Brunner, 2012, chapter 5). One fundamental question is the relationship between population growth, demographic development, and public transport infrastructure. Bogotá, in particular, is going through a key decisionmaking moment regarding the possibility of incorporating a metro system as one of its leading forms of transport. In contrast, the current mass-transit system, Transmilenio, operates using articulated buses. There is a latent need to respond to the question: under what conditions could a mass-transport system invade, expand in the market, and coexist with current, established city transport systems, in the long term? This type of question is closely related to others studied from the standpoint of evolutionary biology, and which have permitted the development of adaptive dynamics as a useful mathematical theoretical framework for the study of these questions.

The formation of new species, called speciation, is one of the central points of evolutionary theory. It occurs through the genetic and phenotypic divergence of populations of the same species, which adapt to different environmental niches, either within the same, or in different habitats. In allopatric speciation, two populations are geographically separated by natural or artificial barriers, while in parapatric speciation, the two populations evolve toward geographic isolation, through the exploitation of different environmental niches in contiguous habitats. In either of these two cases, geographical isolation constitutes 
an exogenous cause of speciation, instead of an evolutionary sequence (Dercole \& Rinaldi, 2008, pp. 67-70; Butlin, Galindo \& Grahame, 2008, p. 2997).

On the other hand, sympatric speciation considers a population in a single geographical location. As such, it is disruptive selection that exerts selection pressures, which favor extreme characteristics over average characteristics. This phenomenon may result, for example, from competition for alternative environmental niches, in which specializing may be more advantageous than being a generalist. Consequently, the population divides into two groups which are initially similar, but which later diverge on separate evolutionary paths (branches), each driven by their own mutations, undergoing what is called evolutionary branching. In figure 1 , the evolutionary branching point concept, a product of sympatric speciation, is shown (Butlin et al., 2008, p. 2997; Doebeli \& Dieckmann, 2000, pp. S77-S78).

Figure 1. Influenced by disruptive selection, a monomorphic population may become dimorphic in certain relevant attributes

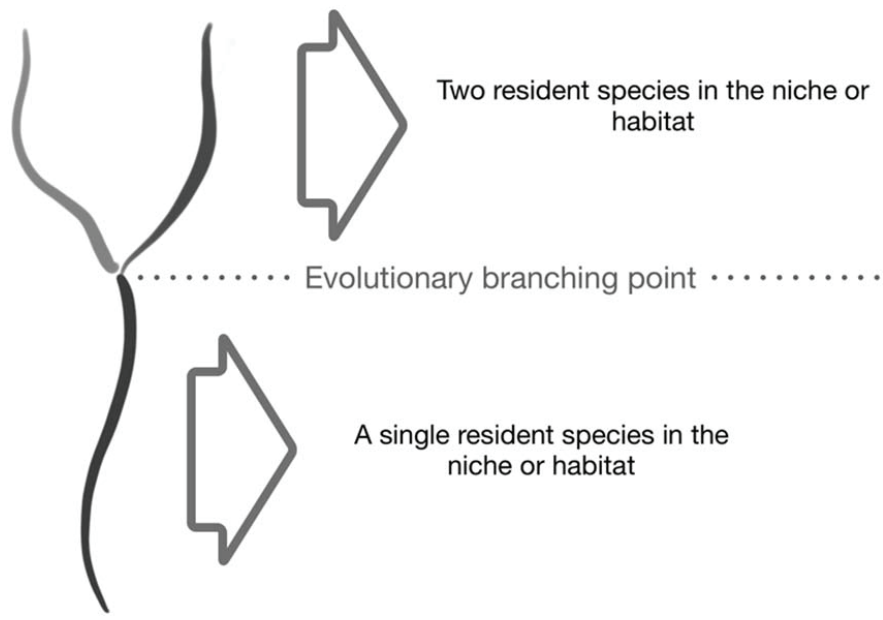

S o u r c e : own study.

Human evolution shows empirical evidence of this evolutionary phenomenon. Humans form part of the hominidae family, which includes great apes (bonobos, chimpanzees, gorillas, and orangutans) and other extinct humanoid species. Since Darwin and the publication of The Descent of Man (1871), countless fossils have been found and dated, which show that humans and great apes 
shared a common ancestor approximately six or seven million years ago. The causes of the evolutionary branching which led to humans are a source of great debate. However, one of the most intriguing potential causes is the evolution of articulated language, thanks to fine control of the larynx or the mouth, which is regulated by a particular gene (Dercole \& Rinaldi, 2008, p. 24; Lai, Fisher, Hurst, Vargha-Khadem \& Monaco, 2001, p. 522).

Generally speaking, the basic units capable of evolution through innovation and competition processes are not limited to living organisms. Multiple examples of evolutionary branching can, in fact, be found in material products, ideas, and social norms (Dercole \& Rinaldi, 2008, p. 33; Dercole, Dieckmann, Obersteiner \& Rinaldi, 2008, p. 335; Dercole, Prieu \& Rinaldi, 2010, p. 380; Landi $\&$ Dercole, 2016, p. 185). In particular, commercial products are replicated each time that a product is bought, and services each time they are used. They go extinct whenever they are abandoned by users. Thus, improved versions are occasionally introduced, which are characterized by small innovations. These interact in the market with the prior, established versions. Said interactions are usually competitive, and involve rivalry between products from both the same and different categories.

One example of this is shown in figure 2, in which the evolution of different communication services in Switzerland, between 1980 and 2000, is shown. The arrival of digital telephones was a successful innovation, which has led to the substitution of analog telephones. This phenomenon is reported as attribute substitution. On the other hand, observe that internet hosts (circles) seem to coexist with both digital telephones and digital mobile telephone service subscribers (Dercole \& Rinaldi, 2008, p. 36).

With the information discussed up to this point, it is possible to respond to the question of what constitutes the theory of adaptive dynamics. In general, it is a theoretical background which originates in evolutionary biology, and links demographic dynamics to evolutionary changes. It further permits the description of evolutionary dynamics in the long term, considering innovations to be small and rare events (Dercole \& Rinaldi, 2008, pp. 67-68; Dieckmann \& Law, 1996, pp. 16-17; Geritz, Metz, Kisdi \& Meszéna, 1997, p. 1; Geritz, Meszéna \& Metz, 1998, p. 1). This theory focuses on the evolutionary dynamic of quantitative adaptation attributes in the long term, and disregards genetic details, through the use of asexual demographic models. Among the most relevant aspects is that it recognizes interactions as the driving evolutionary force, and considers feedback between evolutionary change and the forces of selec- 
tion experienced by agents (Dercole \& Rinaldi, 2008 pp. 67-68; Dercole \& Rinaldi, 2010, pp. 3-4; Doebeli \& Dieckmann, 2000, p. S78).

Figure 2. Use of telecommunication services in Switzerland

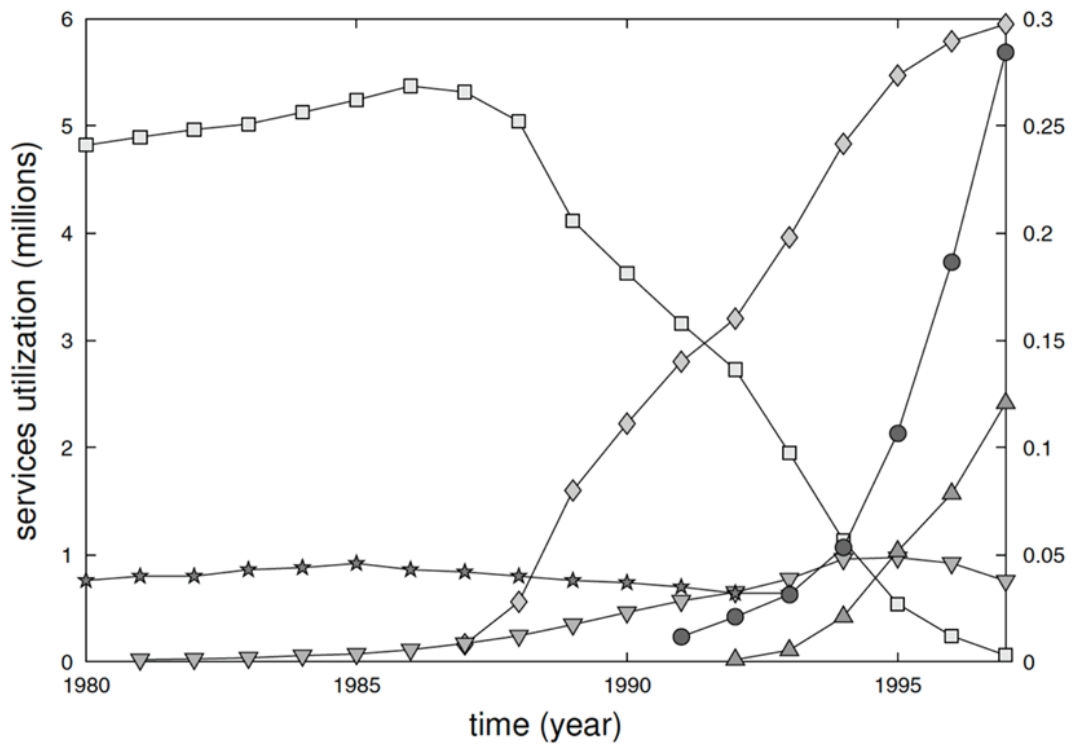

Squ ar e s: analog telephones.

D i a m o n d s : digital telephones.

Inverted triangles: subscribers to analog mobile phone services.

Tria ngle s: subscribers to digital mobile phone services.

St a r s : public payphones.

Circles: internet hosts.

S o u r c e : reprinted from (Dercole \& Rinaldi, 2008, p. 36).

THE RESEARCH METHODOLOGY AND THE COURSE OF THE RESEARCH PROCESS

In this investigation, the question of whether conditions exist for the origin of diversity in a competitive market, among the principal public transport systems (TS) in a city, is addressed from the perspective of adaptive dynamics. Additionally, the average number of passengers transported per unit is considered to be a characteristic attribute of each TS. 
In chapter 3 a model for a stablished TS is proposed, and the local stability is studied. In chapter 4 the model in reformulated to include a technological innovation and a new innovative TS, which allows for determination of the innovative TS fitness function. Invasion conditions are established therefrom in a market dominated by a conventional TS. Later, in chapter 5, based on theory, the canonical equation of adaptive dynamics, which reveals the long-term behavior of the characteristic attribute and its impact on the TS market, is determined and studied. A scenario, in which evolutionary branching occurs, is simulated. This phenomenon is called the origin of diversity, as it implies that the market can be diversified. On the other hand, a scenario in which terminal points occur during attribute evolution, in the case that diversification is not possible, is also presented. Finally, conclusions and references are shown.

\section{A MODEL FOR AN ESTABLISHED TRANSPORT SYSTEM}

Consider a city with an established transport system, which is characterized by a particular attribute, $u_{1}$, which is assumed to be positive and associated with the average number of passengers who are transported in each mobile unit. Denote $x_{1}=x_{1}(t)$, with $0 \leq x_{1} \leq 1$ the proportion of people who adopt the transport system characterized by attribute $u_{1}$. In order to create a model which describes transport system expansion, and which considers the use of the budget allocated for new user acquisition, a reformulation of the user-resource model studied by (Núñez-López, Velasco-Hernández \& Marquet, 2014, p. 3305) is employed:

$$
\dot{x}_{1}=\left[\alpha\left(u_{1}\right) y_{1}-\delta\left(u_{1}\right)\right]\left(1-x_{1}\right) x_{1}
$$

where $\alpha\left(u_{1}\right)$ is the rate of instant TS adoption, and $\delta\left(u_{1}\right)$ is the rate at which the TS is abandoned by its users, such that $\left[\alpha\left(u_{1}\right) y_{1}-\delta\left(u_{1}\right)\right]$ describes the intrinsic rate of TS adoption. In this case, the $y_{1}=y_{1}(t)$ state variable represents the proportion of the budget invested for TS expansion, such that $0 \leq y_{1} \leq 1$. In order to establish the $y_{1}$ budget dynamic, one must consider that the investment cannot be unlimited. Thus, it is assumed that the investor places resources in direct proportion to the unallocated budget $1-y_{1}$, with a $l\left(u_{1}\right)$ proportionality rate. Finally, the budget is invested in magnitude $\epsilon\left(u_{1}\right) \alpha\left(u_{1}\right) y_{1} x_{1}$, in which function $\epsilon\left(u_{1}\right)$ denotes the efficiency with which $y_{1}$ resources are "converted" into new users. Thus, the budget equation is formed as follows: 
$\dot{y}_{1}=l\left(u_{1}\right)\left(1-y_{1}\right)-\epsilon\left(u_{1}\right) \alpha\left(u_{1}\right) x_{1} y_{1}$.

The equations for $\dot{x}_{1}$ and $\dot{y}_{1}$ conform a 2-dimension system, called a resident system, and is given explicitly by the equations:

$$
\begin{aligned}
& \dot{x}_{1}=\left[\alpha\left(u_{1}\right) y_{1}-\delta\left(u_{1}\right)\right]\left(1-x_{1}\right) x_{1} \\
& \dot{y}_{1}=l\left(u_{1}\right)\left(1-y_{1}\right)-\epsilon\left(u_{1}\right) \alpha\left(u_{1}\right) x_{1} y_{1}
\end{aligned}
$$

defined in the square $\Omega=\left\{x_{1}, y_{1} \in \mathbb{R}: 0 \leq x_{1} \leq 1 \wedge 0 \leq y_{1} \leq 1\right\}$. In table 1 , find a description of state variables and of the coefficients used in the resident system.

Table 1. Description of state variables and of the coefficients used in the model

\begin{tabular}{|l|l|}
\hline \hline \multicolumn{2}{|l|}{ State description } \\
\hline \hline$x_{i}$ & Proportion of people who use system $i$ \\
\hline$y_{i}$ & Proportion of the budget available to the expansion of system $i$ \\
\hline \multicolumn{1}{|c|}{ Parameter description } \\
\hline$u_{i}$ & Value of the characteristic attribute which describes TS $i$ \\
\hline$\alpha\left(u_{i}\right)$ & Rate of instant TS $i$ adoption \\
\hline$\delta\left(u_{i}\right)$ & Rate at which TS $i$ is abandoned by users \\
\hline$l\left(u_{i}\right)$ & Rate of investment in new resources for the expansion of TS $i$ \\
\hline$\epsilon\left(u_{i}\right)$ & TS $i$ efficiency of “converting" the investment into new users \\
\hline$c\left(u_{i}, u_{k}\right)$ & Rate of interaction between systems $i$ and $k$. \\
\hline \hline
\end{tabular}

S o u r c e : own study.

For analysis of the above system, and particularly for its numerical study, certain considerations have been taken into account for model coefficients. It has been assumed that, the proportion at which new resources $l\left(u_{1}\right)=l$ are invested, TS efficiency to "convert" the investment into new users $\epsilon\left(u_{1}\right)=\epsilon$ and the rate at which the TS is abandoned by users $\delta\left(u_{1}\right)=\delta$, are constants. On the other hand, it has been assumed that the rate of instant adoption depends on characteristic attribute $u$, through the function: 


$$
\alpha(u)=a \exp \left(-\frac{1}{2 a_{1}^{2}} \ln ^{2}\left(\frac{u}{a_{2}^{2}}\right)\right)
$$

For a TS characterized by attribute $u$ the $\alpha(u)$, rate makes perfect sense when $x_{1}$ is small, and has no competition from other transport systems (Dercole et al., 2008, p. 342). A maximum of $a$ occurs when $u=a_{2}^{2}$, in order to indicate the value of the attribute which is easiest to absorb. On the other hand, for a transport system with a very low or very high number of users, $\alpha(u)$ tends to cancel out with sensitivity controlled by $a_{1}$. Suppose that $a>0$ and $a_{1}, a_{2} \in \mathbb{R}$ (see figure 3).

Figure 3. Function $\alpha(u)$ chart for parameters $a=1, a_{1}=0.5, a_{2}=22.36$.

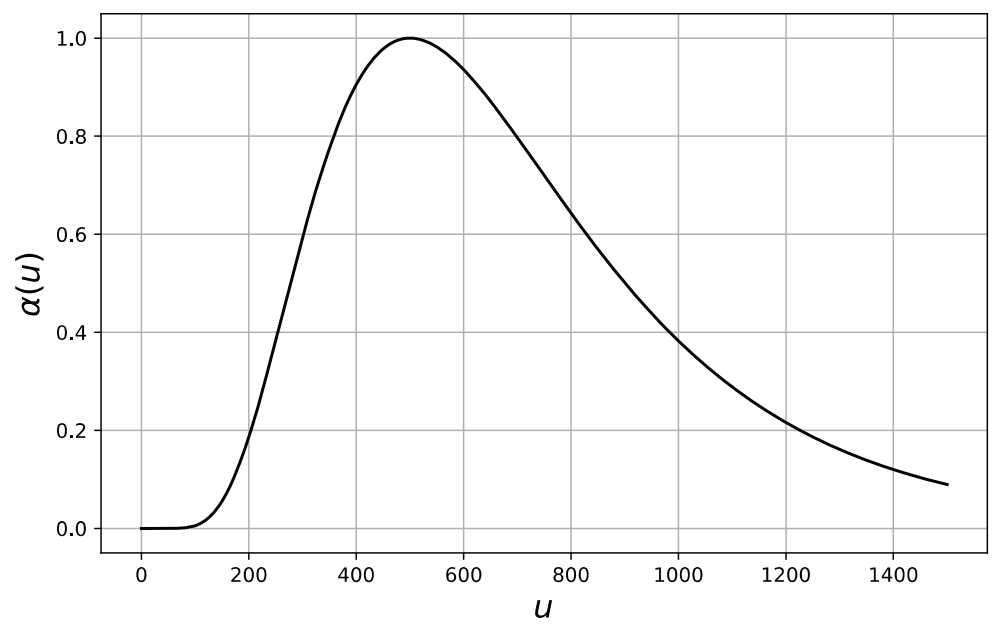

S o u r c e : own study.

Stability analysis is performed through classical methods as described in (Perko, 2013, section 2.6). In this case, the following stationary solutions exist:

- Equilibria $E_{a}=(0,1)$ corresponds to the absence of TS in the city, which in practice, have no interest beyond that which is purely mathematical. However, $E_{a}$ is unstable if, and only if $R_{p}\left(u_{1}\right)>1$, where $R_{p}\left(u_{1}\right)=\frac{\alpha\left(u_{1}\right)}{\delta\left(u_{1}\right)}$. 
- The second equilibria is $E_{0}^{t}=\left(1, \frac{l\left(u_{1}\right)}{l\left(u_{1}\right)+\epsilon\left(u_{1}\right) \alpha\left(u_{1}\right)}\right)$, which corresponds to total TS adoption, or maximum possible adoption.

- The third equilibria is $E_{1}^{p}=\left(\frac{l\left(u_{1}\right)\left(R_{p}\left(u_{1}\right)-1\right)}{\epsilon\left(u_{1}\right) \alpha\left(u_{1}\right)}, \frac{1}{R_{p}\left(u_{1}\right)}\right)$, which corresponds to partial TS adoption by users.

Observe that $R_{p}\left(u_{1}\right)$ represents a growth threshold for the number of transport system users. In effect, $\alpha\left(u_{1}\right)$ is the rate of instant adoption, and is multiplied by $\frac{1}{\delta\left(u_{1}\right)}$, which represents the average amount of time that users employ the TS. Equilibria $E_{1}^{p}$ makes sense if, and only if $0<\frac{l\left(u_{1}\right)\left(R_{p}\left(u_{1}\right)-1\right)}{\epsilon\left(u_{1}\right) \alpha\left(u_{1}\right)}<1$, or similarly, if, and only if:

$$
R_{p}\left(u_{1}\right)>1 \text { and } l\left(u_{-} 1\right)<l^{*}\left(u_{1}\right)=\frac{\alpha\left(u_{1}\right)}{R_{p}\left(u_{1}\right)-1} \epsilon\left(u_{1}\right)
$$

A few important observations regarding the stationary solutions are that, when $R_{p}\left(u_{1}\right), E_{1}^{p}=E_{1}^{a}=(0,1)$ is obtained, and is nonhyperbolic, and when $l\left(u_{1}\right)=l^{*}\left(u_{1}\right), E_{1}^{p}=E_{1}^{t}=\left(1, \frac{1}{R_{p}\left(u_{1}\right)}\right)$ is obtained, it is nonhyperbolic either. In either case, $R_{p}\left(u_{1}\right)=1$ or $l\left(u_{1}\right)=l^{*}\left(u_{1}\right)$, constitute bifurcation values for the resident system with just one TS (Kuznetsov, 2013, chapter 3; Perko, 2013, chapter 4).

Total adoption stationary solution is local, and asymptotically stable (LAS) if, and only if $R_{p}\left(u_{1}\right)>1$ and the investment rate satisfies $l\left(u_{1}\right)>l^{*}\left(u_{1}\right)$. In other words, this is true when user growth is guaranteed and the investment rate is above the minimum required threshold, $l^{*}\left(u_{1}\right)$. Observe that, although system adoption is total, the resources invested are never completely consumed as the equilibria value $0<\frac{l\left(u_{1}\right)}{l\left(u_{1}\right)+\epsilon\left(u_{1}\right) \alpha\left(u_{1}\right)}<1$ is achieved.

Similarly, the equilibria of partial adoption is LAS if, and only if $R_{p}\left(u_{1}\right)>1$ and the investment rate satisfy $l\left(u_{1}\right)<l^{*}\left(u_{1}\right)$. When this equilibria is LAS, the transport system is not being adopted at its total capacity, and consequently, the investment is not completely consumed either.

In table 2, the local stability of stationary solutions is summarized, without considering the previously mentioned extreme cases of bifurcation. 
Table 2. Model local stability results

\begin{tabular}{||cccc||}
\hline Local stability conditions & $E_{1}^{a}$ & $E_{1}^{p}$ & $E_{1}^{t}$ \\
\hline$R_{p}\left(u_{1}\right)<1$ & LAS & $\notin \Omega$ & I \\
$R_{p}\left(u_{1}\right)>1 \wedge l\left(u_{1}\right)<l^{*}\left(u_{1}\right)$ & I & LAS & I \\
$R_{p}\left(u_{1}\right)>1 \wedge l\left(u_{1}\right)>l^{*}\left(u_{1}\right)$ & I & I & LAs \\
\hline
\end{tabular}

S o u r c e : own study.

In figure 4 , line $l\left(u_{1}\right)=l^{*}\left(u_{1}\right)$ is illustrated, explicitly, $l\left(u_{1}\right)=\frac{\alpha\left(u_{1}\right)}{R_{p}\left(u_{1}\right)-1} \epsilon\left(u_{1}\right)$ which splits the $(\epsilon, l)$ - plane into two regions: above the line are points in which the TS achieves a total adoption equilibria, and below the line are points in which the TS achieves a partial adoption equilibria.

Figure 4. Local stability analysis of the resident system

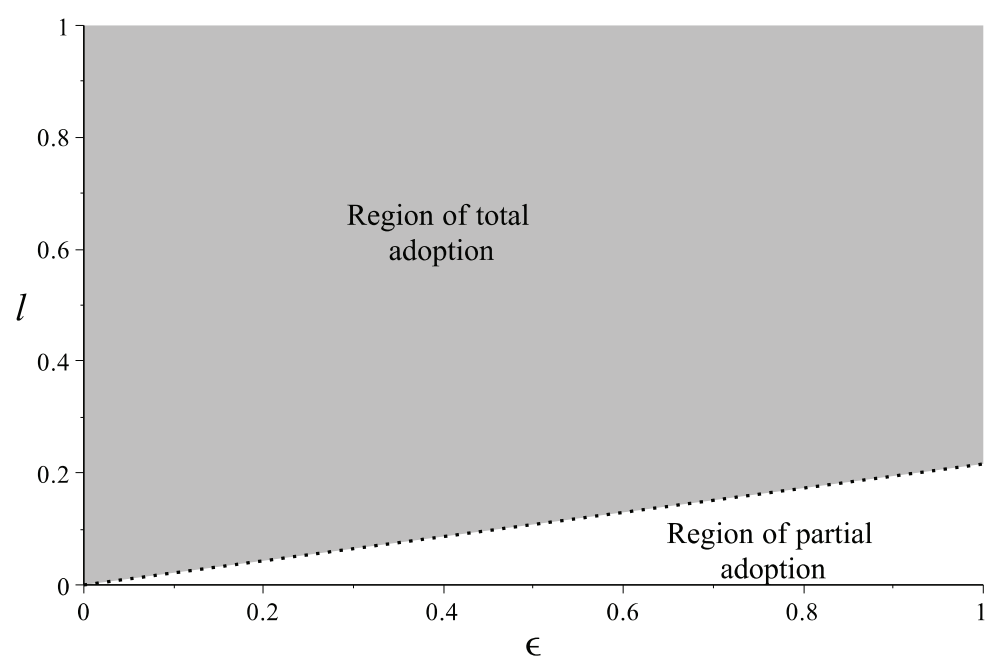

Gray regi on : points in the $(\epsilon, l)$ - plane where equilibria is guaranteed $E_{1}^{t}$ is LAS.

White region: points in the $(\epsilon, l)$ - plane in which equilibria $E_{1}^{p}$ is LAS and $E_{1}^{t}$ is unstable.

The following have been used $\mathrm{a}=1, \mathrm{a}_{1}=0.5, \mathrm{a}_{2}=22.36, \mathrm{~d}=0.1, \mathrm{u}_{1}=200$, and $R_{p}\left(u_{1}\right)=1.8653$.

S o u r c e : own study. 
The values of the parameters used in the simulations (see figure 4), particularly the value of attribute $u_{1}$, is based partially on the capacity of the Transmilenio's bi-articulated buses (massive TS in the city of Bogota, Colombia). They have a 250-person capacity: 62 seated, and 188 standing passengers. Figure 5 shows one of these buses. Considering that the Transmilenio also has smaller buses, the assumption that $\left(u_{1}\right)=200$ has been made. Other parameter values have been established $a d h o c$, and only in order to illustrate model behavior.

Figure 5. Representation of a bi-articulated Transmilenio bus, the mass-transport company in the city of Bogota, Colombia

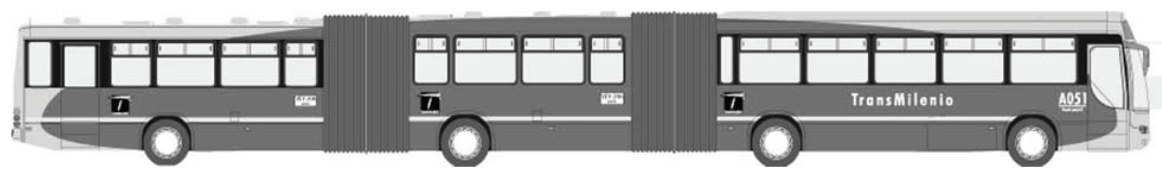

They have a 250-person capacity, 62 seated, and 188 standing passengers.

S o u r c e : reproduced from (www1).

In order to finalize the study of the model with a single established TS, figure 6 shows two simulations which illustrate the two scenarios of interest. In figure 6, $a=1, a_{1}=0.5, a_{2}=22.36, d=0.1$, and $u_{1}=200$ have been used. As such, $l^{*}\left(u_{1}\right)=0.0215$ and $R_{p}\left(u_{1}\right)=1.8653$, with the $l=0.015$ value, and partial adoption is obtained. This is shown in the two boxes on the left, and with $l=0.025$ the total adoption case is obtained, as illustrated in the two boxes on the right. 
Figure 6. Two resident system simulation scenarios are shown for a single TS, with $a=1, a_{1}=0.5, a_{2}=22.36, d=0.1, u_{1}=200, l^{*}\left(u_{1}\right)=0.0215$, and $R_{\mathrm{p}}\left(\mathrm{u}_{1}\right)=1.8653$
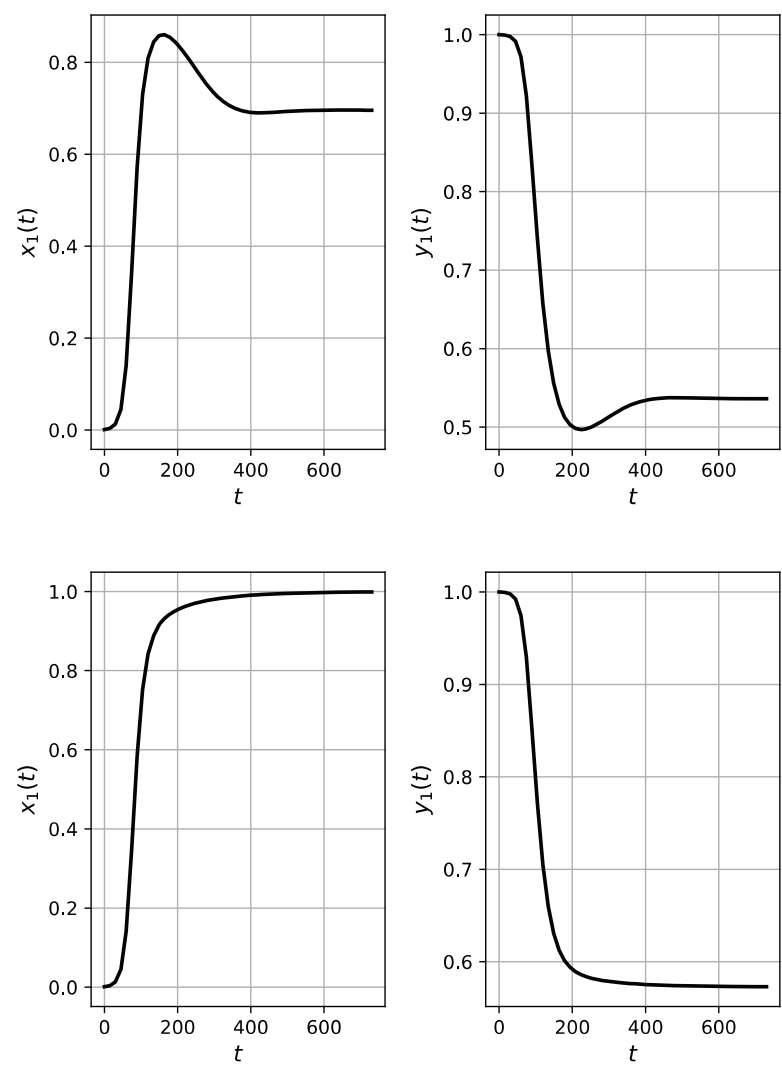

Le f t: solutions for $x_{1}$ and $y_{1}$ are shown, with respect to time for $l=0.015$. As such, the partial adoption equilibria $E_{1}^{p}=(0.70,0.54)$ is LAS.

$\mathrm{Right}$ : the same parameter values are used, but with $l=0.025$. In this case, the total adoption equilibria $E_{1}^{t}=(1,0.57)$ is LAS, is LAS, while $E_{1}^{p} \notin \Omega$.

S o u r c e : own study.

\section{MODEL FOR AN ESTABLISHED AND AN INNOVATIVE TRANSPORT SYSTEM}

Provided that $R_{p}\left(u_{1}\right)>1$, the resident TS system will have, minimally, a nontrivial equilibria, in which it stabilizes. In other words, the partial adoption equilibria $E_{1}^{p}$ or the total adoption equilibria $E_{1}^{t}$ will be LAS. In either case, said equilibria will be denoted by: 


$$
E_{1}=\left(\bar{x}_{1}\left(u_{1}\right), \bar{y}_{1}\left(u_{1}\right)\right)
$$

Suppose that a TS innovation occurs, which corresponds to some technological modification which physically affects the established TS, and leads to the appearance of an innovative TS characterized by the value of attribute $u_{2}$. In general, it is assumed that the innovation is small, and will have a minimal effect, which permits the interaction between transport systems to occur below the same conditions, and on the same market platform. In this case, the difference between the two TSs is in the average number of users they can transport in each mobile unit. The innovative TS gives rise to a small proportion of users $x_{2}=x_{2}(t)$ who compete with the established TS. The success or failure of the innovation may be studied by extending the resident system from the previous section in two more equations: one for $x_{2}$ and another for the corresponding budget $y_{2}$. Explicitly, the fourth-dimension system will take the form:

$$
\begin{aligned}
& \dot{x}_{1}=\left[\alpha\left(u_{1}\right) y_{1}-\delta\left(u_{1}\right)\right]\left(1-x_{1}-c\left(u_{1}, u_{2}\right) x_{2}\right) x_{1} \\
& \dot{y}_{1}=l\left(u_{1}\right)\left(1-y_{1}\right)-\epsilon\left(u_{1}\right) \alpha\left(u_{1}\right) x_{1} y_{1} \\
& \dot{x}_{2}=\left[\alpha\left(u_{2}\right) y_{2}-\delta\left(u_{2}\right)\right]\left(1-x_{2}-c\left(u_{2}, u_{1}\right) x_{1}\right) x_{2} \\
& \dot{y}_{2}=l\left(u_{2}\right)\left(1-y_{2}\right)-\epsilon\left(u_{2}\right) \alpha\left(u_{2}\right) x_{2} y_{2} .
\end{aligned}
$$

This model goes by the name resident - innovative system. Note that, for the model characteristics, must be satisfied that $0 \leq x_{1}+x_{2} \leq 1$. On the other hand, $c\left(u_{i}, u_{k}\right)$ is the interaction rate between systems $i$ and $k$. A number of situations are then obtained:

- If $c\left(u_{i}, u_{k}\right)>1$, inter-system competition prevails over intra-system competition. A simple example of this is that, if system $i$ corresponds to a city taxi system, while system $k$ corresponds to a public bus system, then $c\left(u_{i}, u_{k}\right)>1$ implies that taxi competition with buses is stronger than the competition between the taxis themselves.

- If $c\left(u_{i}, u_{k}\right) \leq 1$, then intra-system competition prevails over inter-system competition. Returning to the public taxi and bus example, in this scenario, competition between the taxis themselves is stronger than competition between taxis and buses. Particularly, $c\left(u_{i}, u_{k}\right)=0$ indicates that there is no interaction between the two TSs, and $c\left(u_{i}, u_{k}\right)=1$ indicates that the interaction between the two TSs is symmetrical, or affects both equally. 
- If $c\left(u_{i}, u_{k}\right)<0$ the interaction between transport systems does not correspond to competition, but rather cooperation, a situation which can describe integrated TSs.

At the time in which innovation occurs, the city established TS was in $E_{1}$ equilibria. In other words, it is assumed that this equilibria is LAS, and as such, the Jacobian matrix denoted here $J\left(E_{1}\right)$ has two eigenvalues with a real negative part. When the resident - innovative system is studied, it may be of interest to determine the conditions under which the innovative TS of attribute $u_{2}$ can "invade" the market. For this, stability conditions at the equilibria:

$$
E_{2}=\left(\bar{x}_{1}\left(u_{1}\right), \bar{y}_{1}\left(u_{1}\right), 0,1\right)
$$

must be studied. The zero and one values in the last two coordinates of $E_{2}$ indicate that the innovative TS has not yet entered the market, and that the entirety of the budget is available for investment. In order to determine local stability, a small disruption is created around it, and the behavior of the linear system associated is studied (Perko, 2013, section 2.6). The corresponding Jacobian matrix takes diagonal form in blocks:

$$
\boldsymbol{J}\left(E_{2}\right)=\left[\begin{array}{ccc}
\boldsymbol{J}\left(E_{1}\right) & \cdots & \\
\mathbf{0} & \delta\left(u_{2}\right)\left(R_{p}\left(u_{2}\right)-1\right)\left[1-c\left(u_{2}, u_{1}\right) \bar{x}_{1}\right] & 0 \\
& -\epsilon\left(u_{1}\right) \alpha\left(u_{1}\right) \bar{y}_{1} & -l\left(u_{2}\right)
\end{array}\right]
$$

where $J\left(E_{1}\right)$ is a $2 \times 2$ matrix, with both of their eigenvalues with real negative parts. Indeed, it corresponds to the Jacobian matrix of the resident system at a LAS equilibria. The $\mathbf{0}$ which appears in the bottom left block is a null $2 \times 2$ matrix. Thus, $\boldsymbol{J}\left(E_{2}\right)^{\prime}$ 's eigenvalues are those contributed by $\boldsymbol{J}\left(E_{1}\right)$, together with $\lambda_{1}=-l\left(u_{2}\right)$, which evidently is negative, owing to the positivity of $l(u)$, and finally $\lambda_{2}=\delta\left(u_{2}\right)\left(R_{p}\left(u_{2}\right)-1\right)\left[1-c\left(u_{2}, u_{1}\right) \bar{x}_{1}\right]$, whose sign will depend on the functions that it involves.

The fitness function is defined via the expression:

$$
\lambda\left(u_{1}, u_{2}\right)=\delta\left(u_{2}\right)\left(R_{p}\left(u_{2}\right)-1\right)\left[1-c\left(u_{2}, u_{1}\right) \bar{x}_{1}\right]
$$

Through study of the sign of this function for specific $u_{1}$ and $u_{2}$ the possibility of innovative transport system invasion may be established. 
In order to numerically study the previous system, it is considered anew that the proportion in which new resources are invested for transport system expansion is $l\left(u_{1}\right)=l$, that the TS efficiency to "convert" the investment into new users is given by $\epsilon\left(u_{i}\right)=\epsilon$, and that the rate at which the TS is abandoned by users $\delta\left(u_{1}\right)=\delta$ are constants for $i=1,2$. Additionally, the interaction rate between TSs is represented in the following form:

$$
c\left(u_{1}, u_{2}\right)=\exp \left(\frac{\ln ^{2} f_{1}}{2 f_{2}^{2}}\right) \exp \left(-\frac{1}{2 f_{2}^{2}} \ln ^{2}\left(\frac{f_{1} u_{1}}{u_{2}}\right)\right)
$$

Observe that the interaction rate between TSs $c\left(u_{1}, u_{2}\right)$ depends on the $\frac{u_{1}}{u_{2}}$ reason, and tends toward zero when said radius tends toward zero, or when it tends toward infinity, which reflects that TSs which are very different compete weakly. A graphic representation of the function is shown in figure 7 (Dercole et al., 2008, p. 342).

Figure 7. Chart of function $c\left(u_{1}, u_{2}\right)$, for parameters $f_{1}=0.96$ y $f_{2}=2$

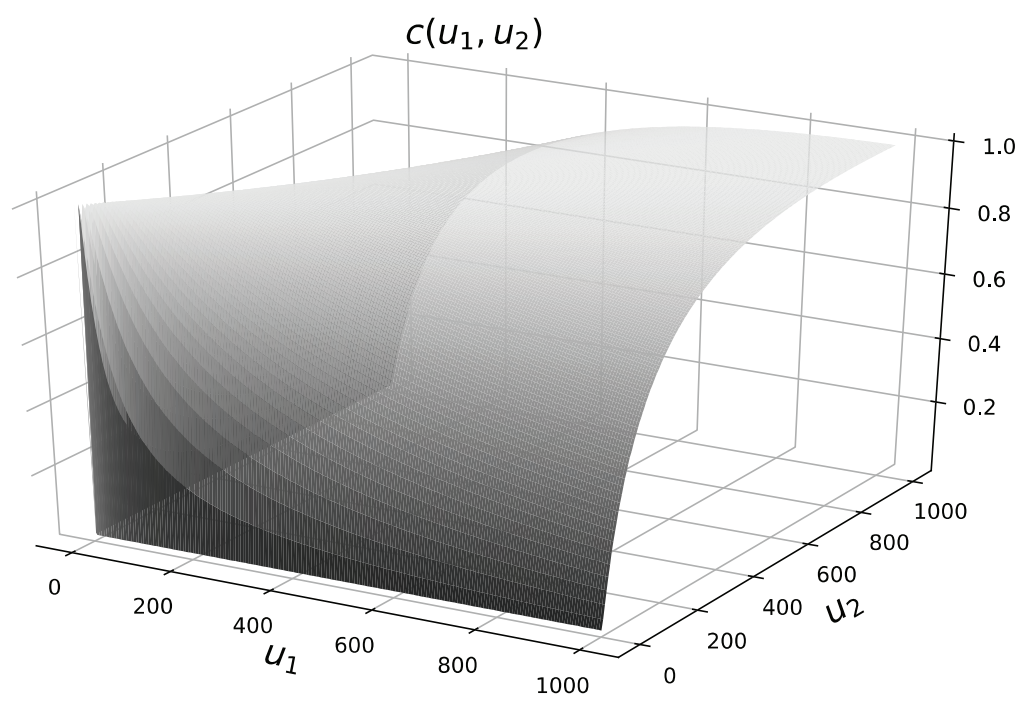

S o u r c e : own study. 
If $f_{1}>1$, the TSs that move the greatest average of passengers tend to have a competitive advantage. On the other hand, if $0<f_{1}<1$, the TSs that move a lower average number of passengers will be those which have the advantage. A large $f_{2}$ value implies that very different TSs also compete strongly. When $f_{1}=1$ competition between TSs is symmetrical.

Figure 8. Representation of a train used in the metro in Medellin, Colombia

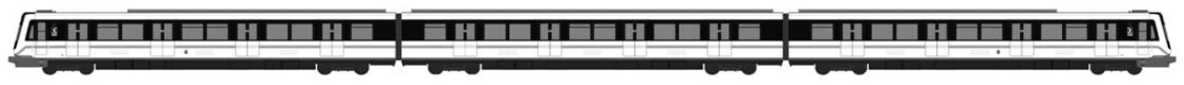

They have a 1220-passenger capacity (eight passengers per square meter), with 148 seats.

S o u r c e : reproduced from (www2).

The values selected for simulations correspond to the belief that innovation involves a TS that is in conditions to transport a higher number of passengers. For this reason, it has been assumed that the value of the established TS attribute is $u_{1}=200$, as stated in the previous section, and that the value of the innovative TS attribute is $u_{2}=800$ Although this value is far above the current capacity of the Transmilenio's bi-articulated buses, it is well below the capacity of other mass TSs. For example, a three-car train from the Medellin, Colombia metro has the capacity to transport up to 1220 passengers at a time. In figure 8 , one of these vehicles is shown.

In figure 9, a simulation of the transport systems is shown, both before and after innovation. The curve shown as a dashed line is the simulation of the resident system before the innovation, respectively, for the $x_{1}$ proportion of users (left), and for the proportion of budget $y_{1}$ (right). Once the innovation occurs, the innovative TS enters the market (dash-dot line), which competes with the established TS (solid line). Observe that this scenario corresponds to diversification, or what here has been called the origin of diversity. Indeed, initially, there was just one TS established in the market. After the innovation, however, both TSs can expand and coexist in the market as two transport options for users. In the figure 9 caption, the values of the parameters used are described. In particular, it should be noted that $f_{1}=0.96$ implies that the TS which mobilizes a lower number of passengers has the competitive advantage. However, innovative transport is able to expand and establish itself in the market. 
Figure 9. Diversification
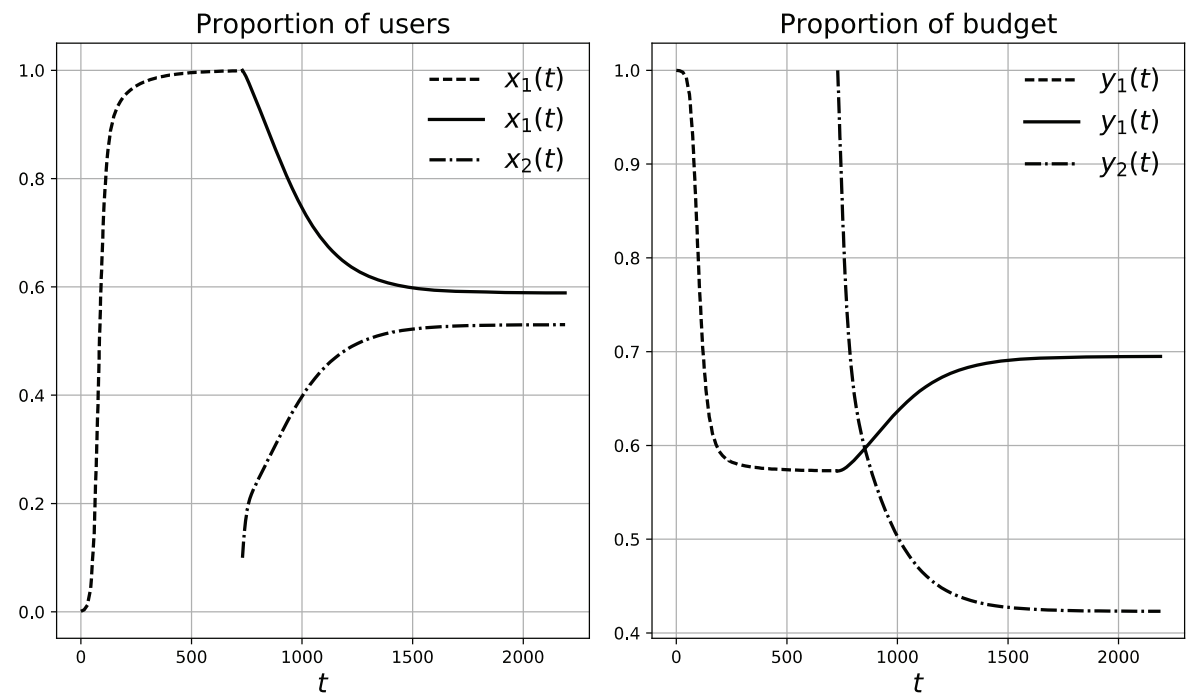

L e f $\mathrm{t}: x_{1}$ solutions before innovation (dashed line) and after innovation (solid line) and for $x_{2}$ (dashdot line). Right: $y_{2}$ solutions before innovation $y_{2}$ (dashed line) and after innovation (solid line) and for $y_{1}$ (dash-dot line). The following have been used: $a=1, a_{1}=0.5, a_{2}=22.36, \delta=0.1, \epsilon=0.1$, $l=0.025, f_{1}=0.96$, and $f_{2}=2$. The attributes $u_{1}=200$ and $u_{2}=800$, thus, $l^{*}\left(u_{1}\right)=0.0215$, $I^{*}\left(u_{2}\right)=0.01184, R_{\mathrm{p}}\left(u_{1}\right)=1.8653$, and $R_{\mathrm{p}}\left(u_{2}\right)=6.428$.

S o u r c e : own study.

Similarly, in figure 10, a substitution scenario is shown. Again, in the dashed line curve, the simulation for the resident system, prior to innovation, is shown, while after the innovation, resident - innovative system solutions are shown using a solid line for $x_{1}$ and $y_{1}$ solutions, and a dash-dot line for the $x_{2}$ and $y_{2}$ solutions. Observe that, after innovation, the innovative TS expands in the market, and is able to substitute the established TS, which tends to have a proportion of zero users in finite time. The only variation that has been performed with respect to the simulation of figure 9 is the $f_{2}=2$ value, which indicates that, in the market, the TS with the capacity of transporting a higher number of passengers is favored. 
Figure 10. Substitution
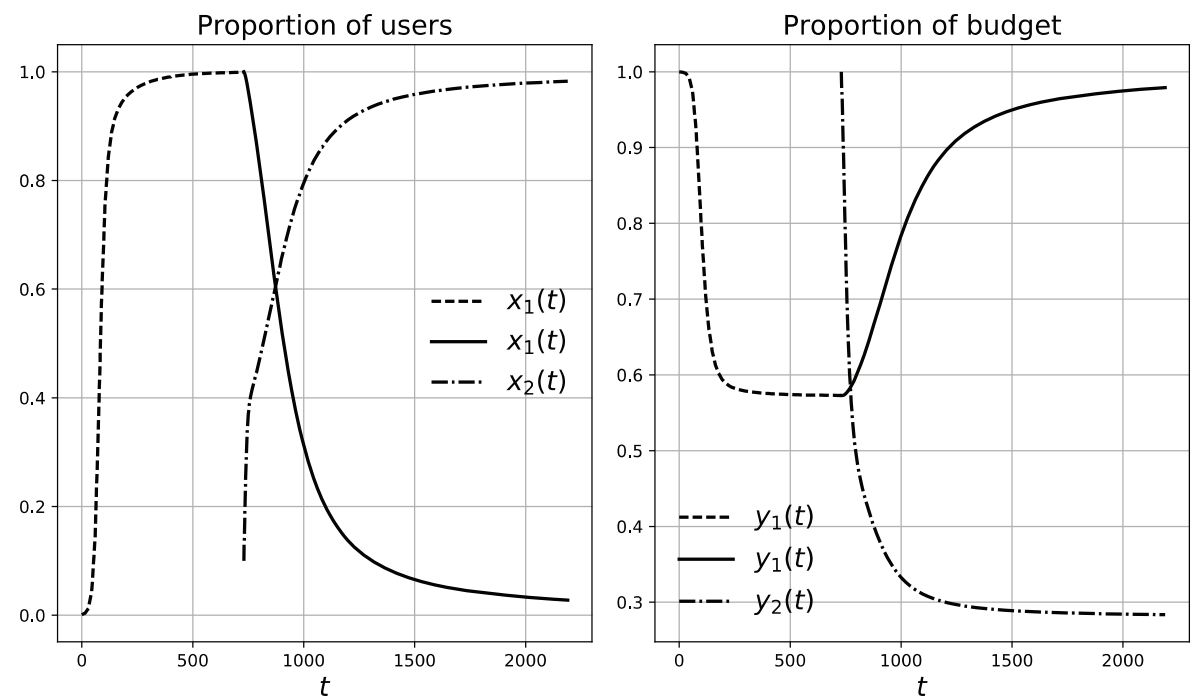

Le f t : $x_{1}$ solutions before innovation (dashed line) and after innovation (solid line) and for $x_{2}$ (dash-dot line). Right: $y_{1}$ solutions before innovation (dashed line) and after innovation (solid line) and for $y_{2}$ (dash-dot line). The same values have been used for parameters, except $f_{1}=2$.

S o u r c e : own study.

\section{THE CANONICAL EQUATION OF ADAPTIVE DYNAMICS}

The dynamic of the attributes, henceforth called the evolutionary dynamic, will help to explain the characteristics of the innovation and competition process which acts on the market. Dercole et al., 2008, chapter 3 , succinctly describes the processes which should be considered for rigorous formulation of the $\mathrm{ca}$ nonical equation, which describes the evolutionary behavior (in the long term) of attribute $u$. This equation takes the general form:

$$
\dot{u}=\frac{1}{2} \mu \sigma^{2} \bar{x}(u) \frac{\partial \lambda}{\partial x_{2}}(u, u)
$$

where $\mu$ is innovation frequency, and $\sigma^{2}$ is variance. In other words, the canonical equation considers the frequency with which innovations are presented in 
the public TS market, and the size of the variations obtained in each innovation. The $\bar{x}(u)$ value corresponds to the equilibria in which the established TS stabilizes before the innovation. On the other hand, partial derivative $\frac{\partial \lambda}{\partial x_{2}}(u, u)$, is called the selection gradient, and is associated with the forces of selection which are exerted from the market, by the same TS users, on the long-term dynamic of the characteristic attribute. Specifically, for the present example:

$$
\dot{u}_{1}=-\frac{1}{2} \frac{\mu \sigma^{2} \ln f_{1}}{a 1^{2} f_{2}^{2} u_{1}^{2}}\left(e^{-\frac{1}{2 a_{1}^{2}} \ln ^{2}\left(\frac{u_{1}}{a_{2}^{2}}\right)} a a_{1}^{2}+a \ln \left(\frac{u_{1}}{a_{2}^{2}}\right) e^{-\frac{1}{2 a_{1}^{2}} \ln ^{2}\left(\frac{u_{1}}{a_{2}^{2}}\right)}-a_{1}^{2} \delta\right)
$$

Whose stationary solutions are:

$$
\bar{u}_{1}=a_{2}^{2} \exp \left(a_{1} \sqrt{-2 \ln \left(\frac{d}{a}\right)}\right), \quad \text { and } \bar{u}_{2}=a_{2}^{2} \exp \left(-a_{1} \sqrt{-2 \ln \left(\frac{d}{a}\right)}\right) \text {, }
$$

When an evolutionary equilibria solution $\bar{u}_{i}$ for $i=1$ or 2 is LAS, this means that successive innovations which replace those previous, drive the attribute toward the value of equilibria $\bar{u}_{i}$. It is important to consider that, in the case of diversification, or when, after innovation in the market, both TSs can coexist, each characteristic attribute will be described by a canonical equation like that described previously. The equations which correspond to this situation are not reported here, as the explicit expressions are quite long and do not significantly contribute to the discussion. However, they may be handled via symbolic calculation.

In figure 11, the behavior of characteristic attributes $u_{1}$ and $u_{2}$ are shown, before and after innovation. It is evident that both attributes diverge in their values to different evolutionary equilibria. While the established TS from attribute $u_{1}$ is maintained below 200 passengers per mobile unit, the innovative TS progressively increases its capacity until reaching an average of over 1400 passengers transported. 
Figure 11. The solutions to the canonical equation for $u_{1}$ shown before innovation (dashed line), for $u_{1}$ canonical equations (solid line) and for $u_{2}$ (dash-dot line) after innovation

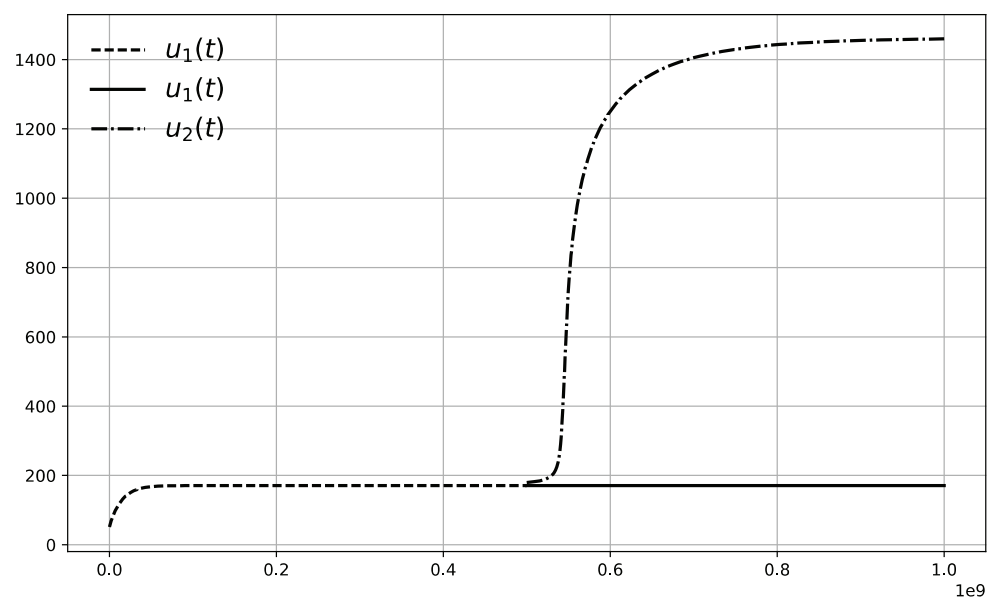

The parameters used are the same as those in figure 9 for the diversification scenario.

S o u r c e : own study.

\section{CONCLUSIONS}

The resident model proposed here is an initial approach to the phenomenon, it permits the study of the dynamics of a city's TS in various scenarios, and learn under which conditions it may be expanded in the market, and a partial or total adoption equilibria could be found, although this would imply transporting the entire population of the city.

One of the features that stand out from the model is that it includes the budget invested to obtain new users as one of the state variables. Based on this consideration, it is possible to obtain explicit conditions under which a equilibrium of partial adoption or one of total adoption of the TS by users can be achieved. In addition, according to this model, both cases are perfectly achievable without consuming all the available resources. In fact, the model made it possible to establish explicit conditions for the level of investment required in each case, information that may be useful in decision making.

The innovative-resident model allows for the establishment of the conditions under which an innovative TS can invade and expand in the market. This information is obtained from study of the sign of the fitness function for spe- 
cific model coefficient expressions. Additionally, the approach through adaptive dynamics permits establishment of the long term dynamics the quantitative attribute (average number of passengers per mobile unit). The study of this evolutionary dynamic permits the classification of the evolutionary equilibrium in ramification points (diversification) or terminal points (those in which the evolution definitively halts), like the points where substitution takes place.

Particularly in the case of diversification, with the coefficients defined in this study, and for the values of the parameters considered, it was observed that the established TS should maintain a low number of users transported ( $<200$ passengers per unit), while the innovative TS should attain a high number of users transported ( $>1400$ passengers per unit).

The above indicates that, in a scenario of coexistence between the two transport systems, it is necessary for each one of them to use a different strategy, in regards to the number of passengers that they decide to transport. One of them should focus on mobile units with few passengers, while the other system should focus on mobile units which can transport passengers massively.

Diversification is impossible when both transport systems use the same strategy. For example, if both TSs design a strategy that permits them to transport over 1400 passengers per unit, the effect would be that the innovative TS would absorb all users and substitute the established TS.

\section{ACKNOWLEDGEMENTS}

G. Olivar and H.D. Toro appreciate the financing provided by Colciencias, via the research project entitled, "Modelado y simulación del Metabolismo Urbano de Bogotá D.C." Code number 111974558276.

\section{REFERENCES}

Baccini, P., \& Brunner, P.H. (2012). Metabolism of the anthroposphere: analysis, evaluation, design. Cambridge: MIT Press. http://dx.doi.org/10.1111/j.1530-9290.2012.00558.x.

Butlin, R.K., Galindo, J., \& Grahame, J.W. (2008). Sympatric, parapatric or allopatric: the most important way to classify speciation? Philosophical Transactions of the Royal Society B: Biological Sciences, 363(1506), 2997-3007.

Dercole, F., Dieckmann, U., Obersteiner, M., \& Rinaldi, S. (2008). Adaptive dynamics and technological change. Technovation, 28(6), 335-348.

Dercole, F., Prieu, C., \& Rinaldi, S. (2010). Technological change and fisheries sustainability: The point of view of Adaptive Dynamics. Ecological Modelling, 221(3), 379-387. 
Dercole, F., \& Rinaldi, S. (2008). Analysis of evolutionary processes: the adaptive dynamics approach and its applications. Princeton: Princeton University Press.

Dercole, F., \& Rinaldi, S. (2010). Evolutionary dynamics can be chaotic: A first example. International journal of bifurcation and chaos, 20(11), 3473-3485. http://dx.doi. org/10.1142/S0218127410027829.

Dieckmann, U., \& Law, R. (1996). The dynamical theory of coevolution: a derivation from stochastic ecological processes. Journal of mathematical biology, 34(5-6), 579-612.

Doebeli, M., \& Dieckmann, U. (2000). Evolutionary branching and sympatric speciation caused by different types of ecological interactions. The american naturalist, 156(S4), S77-S101.

Geritz, S.A., Meszéna, G., \& Metz, J.A. (1998). Evolutionarily singular strategies and the adaptive growth and branching of the evolutionary tree. Evolutionary ecology, 12(1), 35-57.

Geritz, S.A., Metz, J.A., Kisdi, É., \& Meszéna, G. (1997). Dynamics of adaptation and evolutionary branching. Physical Review Letters, 78(10), 2024-2027.

Kennedy, C., Stewart, I. D., Ibrahim, N., Facchini, A., \& Mele, R. (2014). Developing a multi-layered indicator set for urban metabolism studies in megacities. Ecological Indicators, 47, 7-15. http://dx.doi.org/10.1016/j.ecolind.2014.07.039.

Kuznetsov, Y.A. (2013). Elements of applied bifurcation theory (Vol. 112). Berlin: Springer Science \& Business Media.

Lai, C.S., Fisher, S.E., Hurst, J.A., Vargha-Khadem, F., \& Monaco, A.P. (2001). A forkheaddomain gene is mutated in a severe speech and language disorder. Nature, 413(6855), 519-523.

Landi, P., \& Dercole, F. (2016). The social diversification of fashion. The Journal of Mathematical Sociology, 40(3), 185-205. http://dx.doi.org/10.1080/0022250X.2016.1200039.

Núñez-López, M., Velasco-Hernández, J.X., \& Marquet, P.A. (2014). The dynamics of technological change under constraints: adopters and resources. American Institute of Mathematical Sciences, 19(10), 3299-3317. http://dx.doi.org/10.3934/dcdsb.2014.19.3299.

Perko, L. (2013). Differential equations and dynamical systems (Vol. 7). Berlin: Springer Science \& Business Media.

(www1) Transmilenio, http://www.transmilenio.gov.co/Publicaciones/nuestro_sistema/Componentes/Infraestructura (accessed: 15.05.2018).

(www2) Metro de Medellín, https://www.metrodemedellin.gov.co/quiénessomos (accessed: 15.05.2018). 